\title{
Prevention of Chronic Experimental Colitis Induced by Dextran Sulphate Sodium (DSS) in Mice Treated with FR91
}

\author{
Valter R. M. Lombardi, ${ }^{1}$ Ignacio Etcheverría, ${ }^{1}$ Iván Carrera, ${ }^{2}$ \\ Ramón Cacabelos, ${ }^{3}$ and Antonio R. Chacón ${ }^{4}$ \\ ${ }^{1}$ Euroespes Biotechnology, Department of Cellular Immunology, A Coruña, Spain \\ ${ }^{2}$ Euroespes Biotechnology, Department of Neuromorphology, A Coruña, Spain \\ ${ }^{3}$ Euroespes, Department of Pharmacogenetics, A Coruña, Spain \\ ${ }^{4}$ Laboratorios Geamed, Department of $R+D$, Madrid, Spain \\ Correspondence should be addressed to Iván Carrera, neuromorfologia@ebiotec.com
}

Received 3 August 2011; Revised 16 October 2011; Accepted 20 October 2011

Academic Editor: Yasushi Okazaki

Copyright ( 2012 Valter R. M. Lombardi et al. This is an open access article distributed under the Creative Commons Attribution License, which permits unrestricted use, distribution, and reproduction in any medium, provided the original work is properly cited.

One of the main treatments currently used in humans to fight cancer is chemotherapy. A huge number of compounds with antitumor activity are present in nature, and many of their derivatives are produced by microorganisms. However, the search for new drugs still represents a main objective for cancer therapy, due to drug toxicity and resistance to multiple chemotherapeutic drugs. In animal models, a short-time oral administration of dextran sulfate sodium (DSS) induces colitis, which exhibits several clinical and histological features similar to ulcerative colitis (UC). However, the pathogenic factors responsible for DSS-induced colitis and the subsequent colon cancer also remain unclear. We investigated the effect of FR91, a standardized lysate of microbial cells belonging to the Bacillus genus which has been previously shown to have significant immunomodulatory effects, against intestinal inflammation. Colitis was induced in mice during 5 weeks by oral administration 2\% (DSS). Morphological changes in the colonic mucosa were evaluated by hematoxylin-eosin staining and immunohistochemistry methods. Adenocarcinoma and cryptal cells of the dysplastic epithelium showed cathenin- $\beta$, MLH1, APC, and p53 expression, together with increased production of IFN- $\gamma$. In our model, the optimal dose response was the $20 \%$ FR91 concentration, where no histological alterations or mild DSS-induced lesions were observed. These results indicate that FR91 may act as a chemopreventive agent against inflammation in mice DSS-induced colitis.

\section{Introduction}

Inflammatory bowel disease (IBD) is a group of chronic conditions of the colon and small intestine, consisting of Crohn's disease (CD) and ulcerative colitis (UC), characterized by acute pain, vomiting, and diarrhea symptoms followed by remission [1]. A single etiology has not been identified, but rather the pathogenesis of IBD is very complex and involves the external environment, genetic makeup, intestinal microbial flora, and immune system [2]. Although new and powerful medical treatments are available, many are biological drugs or immunosuppressants, which are associated with significant side effects, in particular infection and increased risk of malignancy [3], and elevated costs which require optimal medical treatment adjustment. As a result, the need for predicting disease course and response to therapy is essential. Major attempts have been made at identifying clinical characteristics, concurrent medical therapy, and serological and genetic markers as predictors of response to biological agents. Only few reports exist on how mucosal/tissue markers are capable of predicting clinical behaviour of the disease or its response to therapy [4].

Due to its ability of interfering with intestinal barrier function and stimulating local and systemic inflammation, dextran sodium sulfate (DSS) is often used as a mouse model of colitis which can mimic clinical and histological features of IBD with UC characteristics. Several animal models for intestinal inflammation have been developed in recent years. Most commonly, experimental colitis is induced by heparinlike polysaccharide DSS because of its capacity of inducing 


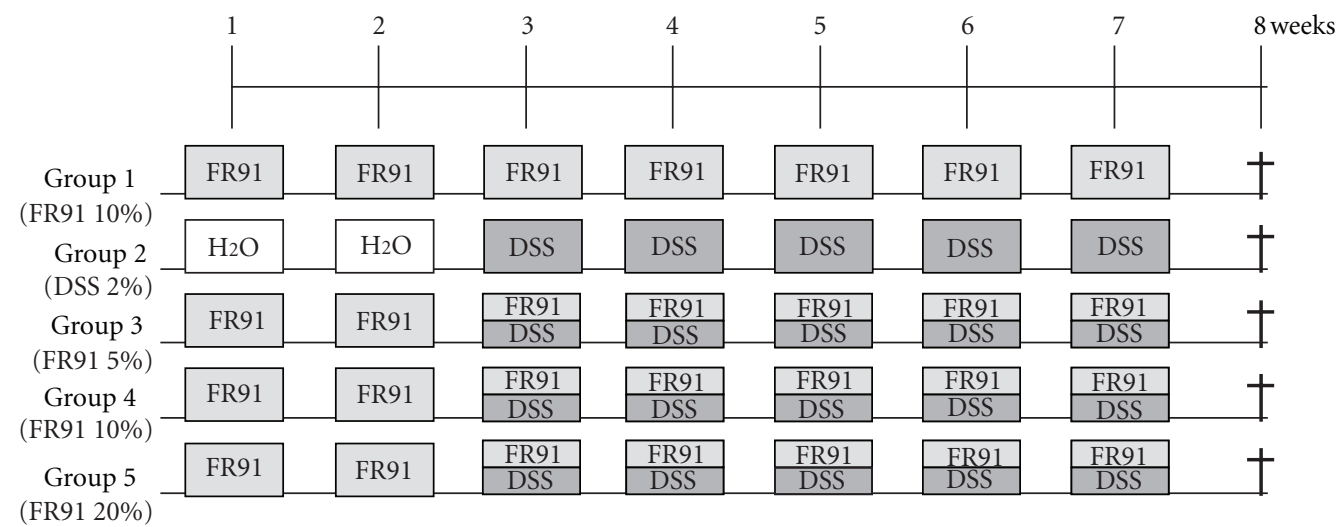

FIGURE 1: Experimental protocol for DSS-induced colitis/colon cancer in mice and the administration of FR91 at different concentrations.

colonic lesions [5]. Okayasu et al. [6] showed that oral administration of $5 \%$ of DSS in drinking water of $\mathrm{BALB} / \mathrm{c}$ mice was able to induce a chronic colitis after several cycles of DSS. This study was followed by a report from Cooper et al. [7] who induced chronic colitis by (a) 7 days of oral DSS followed by 7 days of $\mathrm{H}_{2} \mathrm{O}$ (for 1,2 , and 3 cycles) and (b) 7 days of oral DSS followed by 14 and 21 days of $\mathrm{H}_{2} \mathrm{O}$. The results of this study showed that chronic colitis induced after only 7 days of DSS may serve as a useful model to study the effects of pharmacologic agents in human inflammatory disease and mechanisms of perpetuation of inflammation and gave an extensive description of histological lesions, showing that the main histological changes consisted of focal crypt loss, which was followed by signs of both acute and chronic inflammation.

The present study aimed to investigate the effect of FR91 on the attenuation of the chronic experimental colitis induced by DSS in Swiss mice. Furthermore, we investigated if the chronic phase was characterized by a regulation in the expression of apoptotic genes and by a dysregulation of $\mathrm{T}$ helper 1 (Th1)/T helper 2 (Th2) balance and how this would relate to mucosal regeneration.

\section{Materials and Methods}

2.1. Animals. Twenty-six specific pathogen-free Swiss CD1 female mice (7 weeks old; Santiago de Compostela's University Animal Breeding Core, Spain) were maintained (two or three per cage) in isolator plastic cages with shavings under standard laboratory conditions (sterilizable diet, $50 \%$ humidity, $23-24^{\circ} \mathrm{C}$ temperature, and 12-h light/dark cycle). All mice were quarantined 3 weeks after arrival and then randomized by body weight into experimental and control groups. All mice were permitted free access to a commercial diet and treatment or normal drinking tap water in individual bottles. All procedures conformed to the guidelines established by the European Communities Council Directive of 24 November 1986 (86/609/EEC) and by the Spanish Royal Decree 1201/2005 for animal experimentation and were approved by the Ethical Committee of EBIOTEC.
2.2. Study Design. The design of the present study was focused primarily on inducting colitis-associated dysplasia and/or ulcerative hallmarks by administering synthetic dextran sulfate sodium (DSS) to mice and then treating them with different FR91 (standardized lysate of microbial cells belonging to the Bacillus genus) dilutions as shown in Figure 1. At the age of 7 weeks, the animals were divided into two control groups ( 1 and $2, n=4$ each group) and three experimental groups (3-5, $n=6$ each group). Along the entire experimental procedure, distilled water containing different FR91 dilutions (5\% in group 3,10\% in 1 and 4 , and $20 \%$ in 5) was administrated to the animals in groups $1,3-$ 5. From the third week onwards, distilled water containing $20 \mathrm{~g} / \mathrm{L}(2 \%)$ synthetic dextran sulfate sodium (DSS; mol mass 5000; D4911, Sigma-Aldrich; MO, USA) was also administrated to animals of groups 2-5. For comparison, control groups 1 and 2 received DSS or the FR91 treatment alone, respectively, as untreated control. All mice were sacrificed at the end of the experiment (8th week), at the age of 15 weeks.

2.3. Induction of Colitis. Experimental colitis was induced to mice of groups $2,3,4$, and 5 , by repeated administrations of $2 \%$ (wt $/ \mathrm{vol} ; 20 \mathrm{~g} / \mathrm{L}$ ) DSS in drinking tap water ad libitum for five weeks. This dose was empirically reported to induce moderate to severe colitis while minimize mortality in mice [2]. None of the mice died before the termination of this experiment study at day 50 .

2.4. Tissue Preparation. Mice were deeply anesthetized with ether and intracardially perfused with saline buffer and then fixed by $4 \%$ paraformaldehyde in $0.1 \mathrm{M}$ phosphate buffer $(\mathrm{pH}$ 7.4). The entire colorectum (from colocecal junction to the anal verge) was removed, measured, examined macroscopically, washed with saline buffer, and immediately fixed by immersion in the same fixative for $48 \mathrm{~h}$. Part of the colon was divided into three equal segments (proximal, middle, and distal), and portions were determined under a dissecting stereomicroscope (Leica, M125). Intestine portions were cryoprotected with $30 \%$ sucrose in $0.1 \mathrm{M}$ phosphate buffer, embedded in OCT compound (Tissue Tek, Torrance, CA), and frozen with liquid-nitrogen-cooled isopentane. Parallel 
series of transverse sections of $14-16 \mu \mathrm{m}$ thick were obtained on a cryostat (Starlet 2212, Bright, UK) and mounted on Superfrost Plus (Menzel-Gläser) slides.

2.5. Histological Staining and Immunohistochemistry. Routine histological examination was performed on hematoxylin and eosin (H\&E)-stained sections, where different morphological alterations that occur during inflammationassociated colorectal carcinogenesis (such as cryp abscess, mucosal dysplasia, adenomas, and adenocarcinomas), were identified and diagnosed according to previous published studies, see Keohane et al. [1]. To detect the expression of colorectal histopathological markers such as Catenin- $\beta$, p53, BCL-2, MLH1, and APC, we used immunohistochemical techniques. The sections were pretreated with $\mathrm{H}_{2} \mathrm{O}_{2}$ to eliminate endogenous peroxidase, rinsed twice in phosphatebuffered saline (PBS) at pH 7.4 (10 minutes each), and then sequentially treated with nonspecific binding blocked solution (0.1 M PBS containing 0.2\% Tween 20 and 15\% normal goat serum from Dako; Glostrup, Denmark) for $1 \mathrm{~h}$, primary rabbit policlonal antibodies such as anticatenin- $\beta$, anti-p53, anti-BCL-2, anti-MLH1, and anti-APC antibodies (rabbit antibodies that were affinity purified from rabbit antiserum by affinity chromatography using mouse epitope-specific immunogen; Bioworld Technology, MN, USA; Cat. No. BS3603, BS3736; BS1511, BS2418, BS1017 respectively, dilution $1: 200$ ) overnight, PBS (two 10-min rinses), goat anti-rabbit IgG serum biotinylated (Dako, dilution $1: 100$ ) for $1 \mathrm{~h}$, PBS (two 10-min rinses), Vectastain ABC Kit (Vector Laboratories, Burlingame, CA, USA) for $1 \mathrm{~h}$, and PBS (two 10-min rinses). As a negative control, omission of the primary, secondary or tertiary antibodies were used, and no immunostaining was observed. At the last step, the immunoreaction was developed with $0.005 \%$ diaminobenzidine (DAB; Sigma-Aldrich) and $0.003 \% \mathrm{H}_{2} \mathrm{O}_{2}$. All dilutions were made in PBS containing $0.2 \%$ Tween 20, and incubations were made in a humid chamber at room temperature. Finally, the sections were dehydrated, mounted, and coverslipped.

Antibody characterization and specificity. According to the technical information supplied by the manufacturer (Bioworld Technology, MN, USA), the primary antibodies used were raised against denatured mouse epitopes from rabbit antiserum, and they were affinity purified by chromatography using epitope-specific immunogen with purity higher than 95\% (by SDS-PAGE). Its specificity has been assessed by Western blot; it recognizes a single-protein band of approximately $\approx 86-90 \mathrm{kD}(\beta$-catenin $), \approx 43-45 \mathrm{kD}$ (p53), $\approx 26-28 \mathrm{kD}($ BCL-2), $\approx 84-86 \mathrm{kD}$ (MLH1), and $\approx 270$ $280 \mathrm{kD}$ (APC). Moreover, antibodies have wide species crossreactivity and were used for demonstrating their expression in mouse, rat, and human. Lesions were classified as positive for catenin- $\beta /$ BCL-2/MLH1/APC if cytoplasmic/nuclear staining was detected, and p53 was considered positive if nuclear expression was detected. Two different observers evaluated individually and independently the experimental group slides in a double-blind manner and achieved a high level of concordance. Three sections of each mouse colorectal segment were coded and scored for lesions according to the extent ulceration ( 0 , not present and 1 present), severity of lesions, hyperplasia, and area involved, graded as follow: 0 , normal; 1 , mild; 2 , moderate; 3 , severe.

2.6. Imaging. The sections were photographed with an Olympus microscope (BX50) equipped with a color digital camera (DP10). The photographs were converted to gray scale and adjusted for brightness and contrast with Corel Draw (Corel, Ottawa, Canada), and the plates composed with Corel Photo Paint.

2.7. Th1/Th2 Cytokine Profile Detection. Quantitative detection of GM-CSF, IFN- $\gamma$, IL-1 $\beta$, IL-2, IL-4, IL-5, IL-6, IL10, IL-17, and TNF- $\alpha$ was performed by using a FlowCytomix Mouse Th1/Th2 10plex (BMS820FF) from Bender MedSystems which allowed to measure multiple analytes in a single 50 microliter aliquot of mice sera. In brief, microparticle beads were dyed with differing concentrations of two fluorophores to generate distinct bead sets. Each bead set was coated with capture antibody specific for one analyte. Captured analyte was detected using a biotinylated detection antibody and streptavidin-phycoerythrin (S-PE). A FACScan flow cytometer from Becton Dickinson was used to acquire samples. For calculation of results, the FlowCytomix Pro Software (Bender MedSystems) was used.

\section{Results}

\subsection{Treatment with FR91 Ameliorates Acute and \\ Chronic DSS-Induced Colitis}

3.1.1. Pathological and Inflammatory Findings. The mice that received repeated administrations of $2 \%$ DSS and lower or absent levels of FR91 (groups 2 and 3) showed bloody stools during the second half of the experiment, (from the fifth week onwards), whereas no such internal inflammation feature was observed in the other mice groups. Macroscopically, we have identified numerous gross inflammatory polypoid lesions in mice of groups 2 (6/6; $100 \%)$ and $3(5 / 6 ; 83.3 \%)$, mainly on the middle and distal portions of the colorectal segment, and very few were observed in group $4(1 / 6 ; 16.6 \%)$. Remarkably, none of the mice group 5 (DSS/20\%FR91) showed any ulcer formation in the colorectal segment analyzed, as well as the treatment control mice group $1(10 \%$ FR91) that was also free of colitic ulcerations. These macroscopical observations were confirmed by histological analysis of the intestinal morphology emphasizing the alterations regarding the integrity and inflammation of colonic mucosa and submucosa, dysplastic epithelium, and the presence of ulcers. This histological examination of transverse sections staining with H\&E (Figure 2) showed ulcers with moderate-to-severe morphological alterations (multifocal areas of inflamation in the submucosa or ulcers that covered large mucosal areas), mild-to-severe crypt hyperplasia (lining epithelium was two to three times normal thickness, marked hypercromasia of cells, and multiple crypts with arborizing pattern), epithelial dysplasia (alteration in the differentiation of epithelial cells that may progress to invasive carcinoma), and large affected 


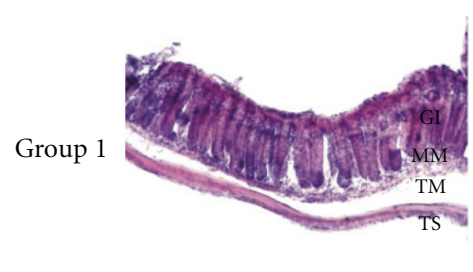

(a)

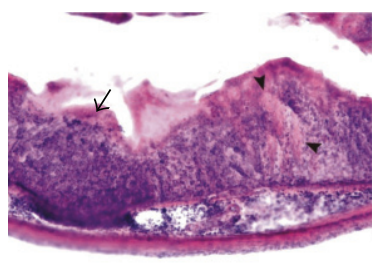

(e)

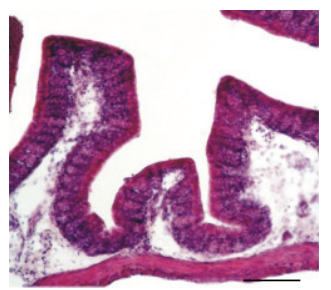

(i)

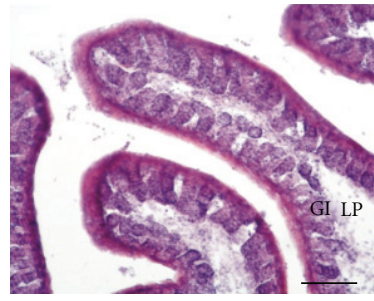

(b)

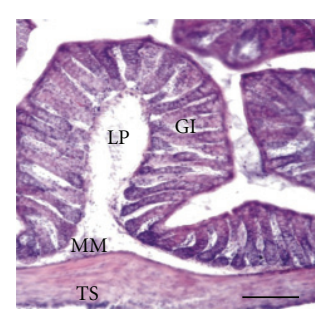

(c)

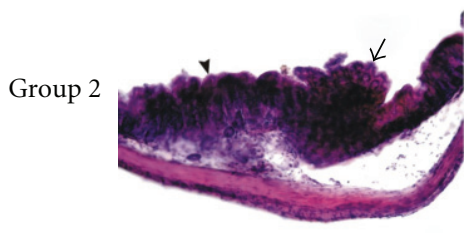

(d)

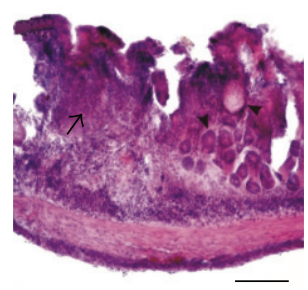

(f)

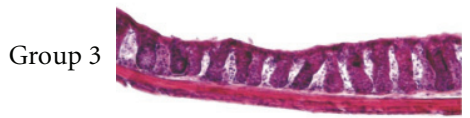

(g)

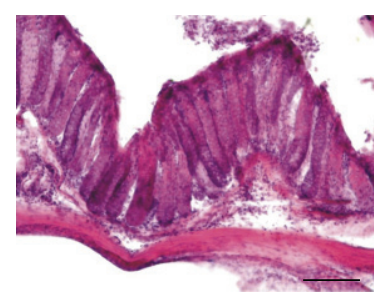

(k)

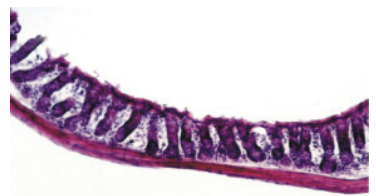

(h)

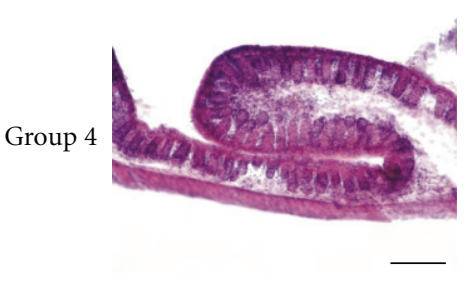

(j)

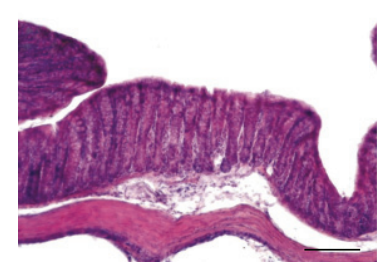

$(\mathrm{n})$

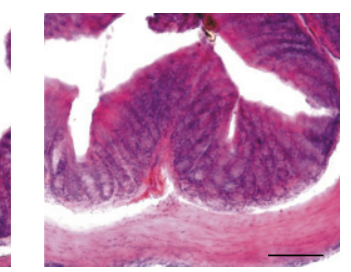

(o)

$(\mathrm{m})$

FIgURE 2: Histological sections of three different portions of the experimental mice colon to show histopathological lesions identified by hematoxylin and eosin staining. (a-c) Transverse sections at three different colorectal levels (proximal, middle, and distal) of mice group 1 treated with FR91 (10\%). Note the absence of colonic lesions and the similar epithelial structures observed in normal control mice. (df) Transverse sections at three different colorectal levels of mice group 2 treated with DSS (2\%) for 5 weeks. In each studied level, multiple histopathological lesions were observed, being particularly clear the severe-grade dysplasia (arrowheads in (d) and (e)) developed in the colon of mice from this group, together with aberrant crypts (arrow in (d)), adenomatous polyps (arrowhead in (f)), and incipient ulcerations (arrow in (f)). (g-I) Transverse sections at three different colorectal levels of mice group 3, with the administration of DSS (2\%) for 5 weeks and treated with FR-91 (5\%). Although these three colonic levels show a low-grade dysplasia and some scattered mucosal ulcerations, the structural histological pattern in general is functional. (j-1) Transverse sections at three different colorectal levels of mice group 4, with the administration of DSS (2\%) for 5 weeks and treated with FR-91 (10\%). This group showed a better colorectal histological organization than that observed in group 3 although there are some mild-grade dysplastic crypts mainly at the distal segments. (m-o) Transverse sections at three different colorectal levels of mice group 5, with the administration of DSS (2\%) for 5 weeks and treated with FR-91 (20\%). These sections show a normal epithelial organization, with well-differentiated cryptal cells and no atypical lesion features. Scale bar: $100 \mu \mathrm{m}$.

areas of crypt loss. However, severity of these colorectal lesions differed significantly among mice groups, being the mice group 2 (DSS) the one with a high severity level, while the mice group 3 (DSS/5\%FR91) showed a mild-tomoderate severe level, and the mice group 4 (DSS/10\%FR91) presented a few mild scattered lesions. No colitis characteristic lesions were observed in the colorectal segment of mice group 5 (DSS/20\%FR91) or in the treatment control mice group 1 (10\%FR91), (Figure 2), as described previously in the macroscopical exam. Histological scoring data obtained from the colorectal lesions examination are presented in Table 1.

3.1.2. Immunohistochemistry of Catenin- $\beta, p 53, B C L-2$, $M L H 1$, and APC. Immunohistochemical techniques used to identify cell markers of the colorectal lesions showed 
TABLE 1: Histological scores for the colorectal lesions observed in five experimental mice groups. Data are expressed as mean \pm standard deviation.

\begin{tabular}{llcccccr}
\hline & $n$ & Colon segment affected & $\begin{array}{c}\text { Extent } \\
\text { ulceration }\end{array}$ & Severity & Hyperplasia & $\begin{array}{c}\text { Area } \\
\text { involved }\end{array}$ & Total score \\
\hline Group 1 & 4 & Normal & 0 & 0 & 0 & 0 & 0 \\
Group 2 & 4 & Proximal, middle, distal & 1 & $2.6 \pm 0.23$ & $2.8 \pm 0.43$ & $3.2 \pm 0.33$ & $9.6 \pm 0.99$ \\
Group 3 & 6 & Middle, distal & 0 & $1.5 \pm 0.18$ & $2.7 \pm 0.31$ & $2.4 \pm 0.17$ & $6.6 \pm 0.66$ \\
Group 4 & 6 & Middle, distal & 0 & $0.6 \pm 0.14$ & $1.1 \pm 0.13$ & $0.9 \pm 0.20$ & $2.6 \pm 0.47$ \\
Group 5 & 6 & Normal & 0 & 0 & 0 & 0 & 0 \\
\hline
\end{tabular}

expression of Catenin- $\beta$ (cellular adhesion regulator), BCL-2 (apoptotic regulator), MLH1 (DNA-mismatch repair), APC, and p53 (tumour suppressor proteins) in all colonic mice lesions observed and described above. Catenin- $\beta$ antibody detected endogenous expression levels of catenin- $\beta$ protein mainly in the cytoplasm of adenocarcinoma and dysplastic cells. Intense catenin- $\beta$ expression was observed in the proximal and middle (Figure $3(\mathrm{f})$ ) colon of mice group 2 (DSS) whereas a moderate-to-intense immunoreactivity was also observed in the colorectal segments (Figure $3(\mathrm{k})$ ) of mice group 3 (DSS/5\%FR91). Catenin- $\beta$-immunoreactive (-ir) cells were localized in the dysplastic criptal cells and adenocarcinoma cells, at the internal criptal layers (Figures $3(\mathrm{f})$ and $3(\mathrm{k}))$. Colorectal portions of mice groups 1,4 , and 5 showed a weak or absent catenin- $\beta$-positive reaction in their cryptal cells (Figures $3(\mathrm{a}), 3(\mathrm{p})$, and $3(\mathrm{u})$ ), considered as catenin- $\beta$ cellular basal expression. BCL-2-ir and p53-ir cells were observed at colorectal portions of mice groups 2 (Figures $3(\mathrm{~g})$ and $3(\mathrm{j})$ ) and 3 (Figures $3(\mathrm{l})$ and $3(\mathrm{o})$ ), showing an intense staining in adenocarcinoma and cryptal cells, particularly strong in mice group 2 . Immunoreactivity to BCL-2 and p53 was absent in colorectal section of mice groups 1,4 , and 5 studied (Figures 3(b), 3(q), 3(v), 3(e), 3(t), and $3(\mathrm{y}))$. Strong staining MLH1-ir cells were observed at colorectal segments of mice groups 2 (Figure $3(\mathrm{~h})$ ) and 3 (Figure 3(m)), showing an intense staining in adenocarcinoma and cryptal cells of the dysplastic epithelium, particularly intense in mice group 2. MLH1-ir cells were absent in colorectal section of mice groups 1,4 , and 5 studied (Figures 3(c), 3(r), and 3(w)). APC immunoreactivity was intensely observed in all colorectal portions of mice groups 2 (Figure 3(i)) and 3 (Figure 3(n)) although cryptal cells of mice group 2 showed a stronger positivity. Colorectal portions of mice groups 1, 4, and 5 showed a weak APC immunoreactivity in their cryptal cells (Figures 3(d), 3(s), and $3(\mathrm{x}))$.

The incidence of colon lesions showed by these cell markers in the group 5 was $8.2 \%( \pm 4 \%)$, whereas it was $38.2 \%( \pm 6 \%), 33.3 \%( \pm 6 \%)$, and $20.4 \%( \pm 3 \%)$ in groups 2,3 , and 4, respectively (see Figures 4 and 5). Similar histological incidence was observed in mice group 5 when compared with negative control group $1(7.8 \%, \pm 2 \%)$. Twofactor ANOVA of data for each immunohistological markers in the colorectal portion showed significant differences among experimental groups $1 / 5$ and 2/3/4, (Figure 5). Group 5 was the most resistant mice group to DSS-induced lessions in the colorectal portion, as indicated by the lowest value for each marker, similar to that observed in the control group 1. Groups 2, 3, and 4 showed more susceptibility to DSSinduced lessions, consistent with high values. Significant group differences were found between group $1 / 5$ and 2/3/4 in DSS susceptibility, being correlated with differences in the FR91 mean consumption per group.

3.1.3. DSS Production of Inflammatory Cytokines. We next considered potential mechanisms which might underlie the colitis exhibited by DSS treatment and the effect of FR-91 in the treated mice groups. As shown in Table 2, DSS treatment increased only the production of IFN- $\gamma$ proinflammatory cytokine. No significant changes were detected in IL- $1 \beta$, TNF- $\alpha$, IL-6, IL-10, and IL-17 in both treated and untreated groups.

\section{Discussion}

More than 20,000 bioactive compounds synthesized by microorganisms have been identified, and over 10,000 of these secondary metabolites are produced by actinomycets, representing $45 \%$ of all bioactive metabolites discovered [8]. Among actinomycetes, around 8,000 compounds are synthesized by Streptomyces species [8]. The main function of these compounds is antibiotic activity. In addition, many of these compounds, such as anthracyclines (aclarubicin, daunomycin, and doxorubicin), peptides (bleomycin and actinomycin D), aureolic acids (mithramycin), enediynes (neocarzinostatin), antimetabolites (pentostatin), carzinophilin, mitomycins, and others $[9,10]$ have also been tested for the inhibition of chemically induced carcinogenesis in both in vitro and in vivo animal models [11].

In the present study, we investigated the effect of FR91, a standardized lysate of microbial cells belonging to the Bacillus genus which has been previously shown to have significant immunomodulatory effects tested on human tumor cell lines [12], on colonic inflammation induced by 8 -week exposure of $2 \%$ DSS in the drinking water and, in particular, whether FR91 affects colorectal inflammation. We found that the six-week treatment resulted in a slightly reduction of colorectal lesions at lower dosis (5\% FR91) and a moderate-to-complete reduction at higher dosis (10-20\% FR91). Moreover, histopathological data showed that FR91 has no pathological effect on the morphological organization of the mice colon tissues, as observed when administering 


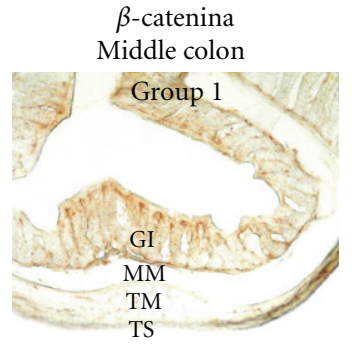

(a)

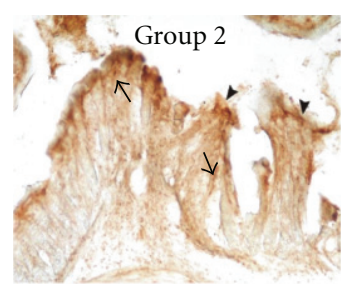

(f)

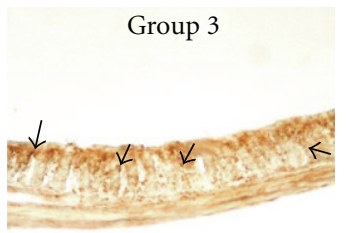

(k)

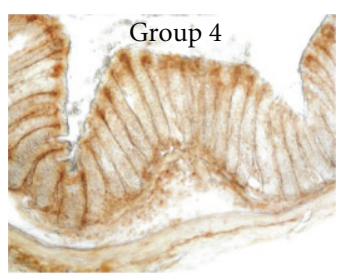

(p)

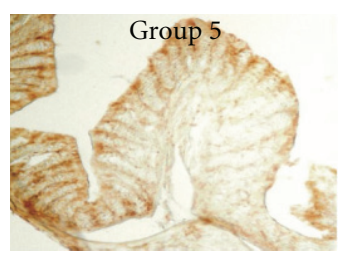

(u)

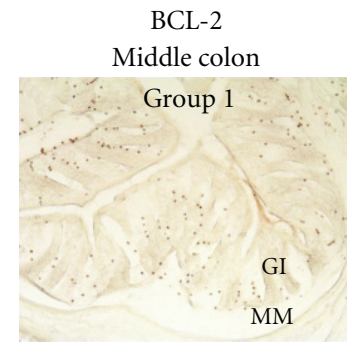

(b)

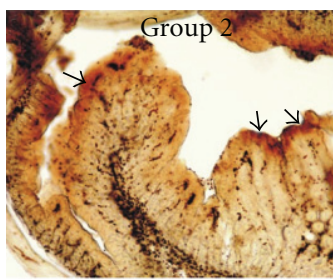

(g)

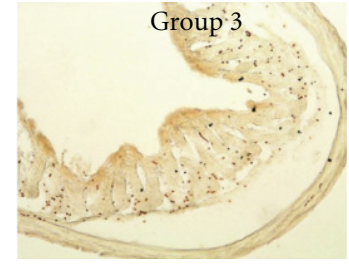

(1)

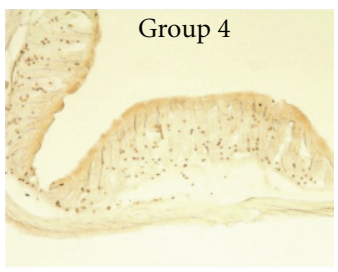

(q)

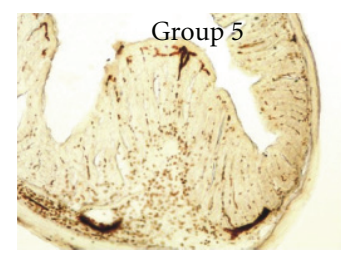

(v)

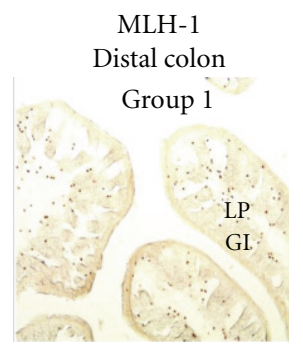

(c)

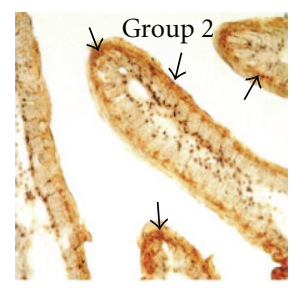

(h)

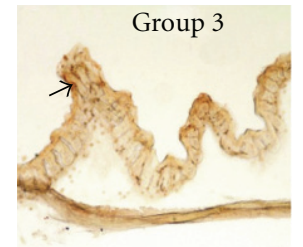

(m)

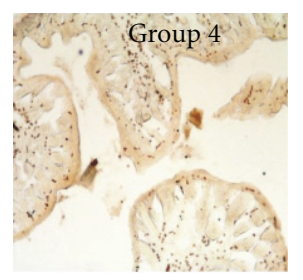

(r)

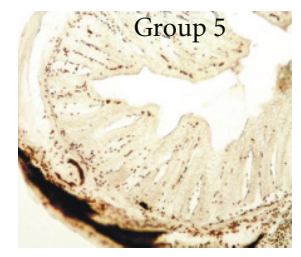

(w)

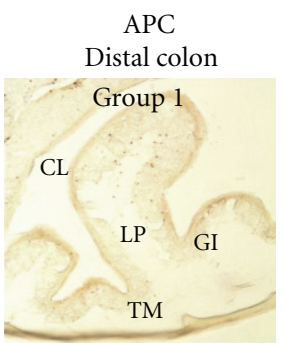

(d)

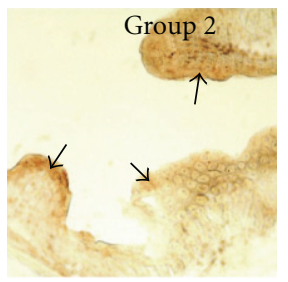

(i)

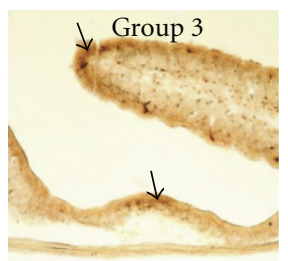

(n)

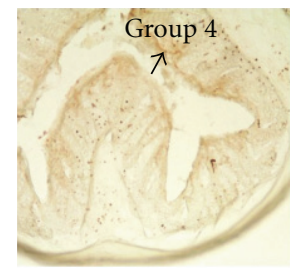

(s)

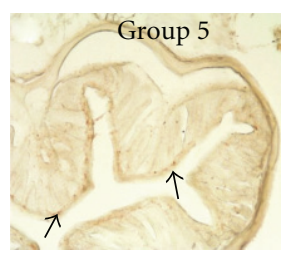

(x) p53

Distal colon

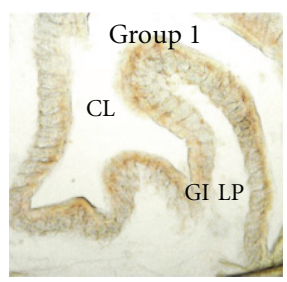

(e)

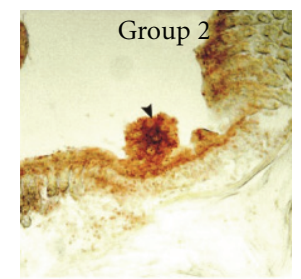

(j)

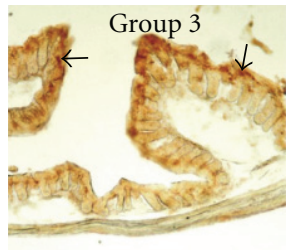

(o)

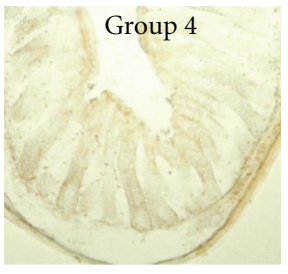

(t)

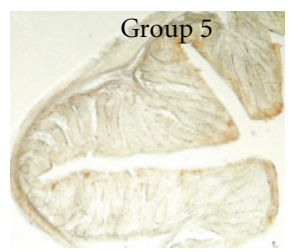

(y)

FIgURE 3: Immunohistochemistry of colorectal pathological markers in different transverse section of colon in mice. (a-e) Details of transverse sections at middle and distal colorectal levels of mice group 1, showing scarce immunoreactivity to Catenin- $\beta$, p53 or absence of BCL-2, MLH-1, and APC. Note that these sections also show a normal epithelial organization with well-differentiated cryptal cells. (f-j) Details of transverse colon sections of mice group 2, showing intense immunoreactivity to the cell markers studied. Note the numerous immunoreactive cells (arrows in (f-i)) and their massive location at the different pathological lesions, such as severe-grade dysplasia (arrowheads in (f)), polyps (arrowhead in (j)), and adenomas. (k-o) Details of transverse colon sections of mice group 3, showing moderateto-scarce immunoreactivity to the cell markers in the colorectal segments. Note some immunoreactive cells to Catenin- $\beta$ (arrowheads in (k)), MLH-1 (arrow in (m)), APC (arrows in (n)), and p53 (arrows in (o)). (p-t) and ( $\mathrm{u}-\mathrm{y})$. Details of transverse colon sections of mice group $4(\mathrm{p}-\mathrm{t})$ and goup $5(\mathrm{u}-\mathrm{y})$, showing absence or basal (arrows in $(\mathrm{s})$ and $(\mathrm{x}))$ immunoreactivity to the cell markers in the colorectal segments studied. Scale bar: $100 \mu \mathrm{m}$. 
TABLe 2: Levels of IL-1 $\alpha$ (pg/mL), IL-2 (pg/mL), IL-5 (pg/mL) IL-6 (pg/mL), IL-10 (pg/mL), IFN- $\gamma$ (pg/mL), TNF- $\alpha$ (pg/mL), GM-CSF $(\mathrm{pg} / \mathrm{mL}), \mathrm{IL}-4(\mathrm{pg} / \mathrm{mL})$ and IL-17 $(\mathrm{pg} / \mathrm{mL})$ in mice serum samples. Data are expressed as mean \pm standard deviation.

\begin{tabular}{lcccccccccc}
\hline & IL-1 $\alpha$ & IL-2 & IL-5 & IL-6 & IL-10 & IFN- $\gamma$ & TNF- $\alpha$ & GM-CSF & IL-4 & IL-17 \\
\hline FR-91 & 0 & 0 & $20 \pm 1.5$ & $25 \pm 2.5$ & 0 & 0 & 0 & $78 \pm 3.8$ & 0 & 0 \\
DSS & 0 & 0 & $78 \pm 4.5$ & $48 \pm 5.4$ & $175 \pm 4.5$ & $363 \pm 13.4$ & $125 \pm 9.7$ & 0 & 0 & $1 \pm 0.2$ \\
$5 \%$ & 0 & $55 \pm 3.3$ & $78 \pm 3.9$ & $53 \pm 7.6$ & $96 \pm 10.1$ & 0 & 0 & 0 & 0 & $24 \pm 2.9$ \\
$10 \%$ & 0 & $34 \pm 3.8$ & $37 \pm 2.1$ & $149 \pm 9$ & $97 \pm 8.6$ & 0 & 0 & $41 \pm 5.7$ & 0 & $12 \pm 1.7$ \\
$20 \%$ & 0 & 0 & $38 \pm 3.9$ & $47 \pm 7.2$ & $55 \pm 5.8$ & $15 \pm 2.1$ & 0 & $75 \pm 4.4$ & 0 & $17 \pm 2.5$ \\
\hline
\end{tabular}

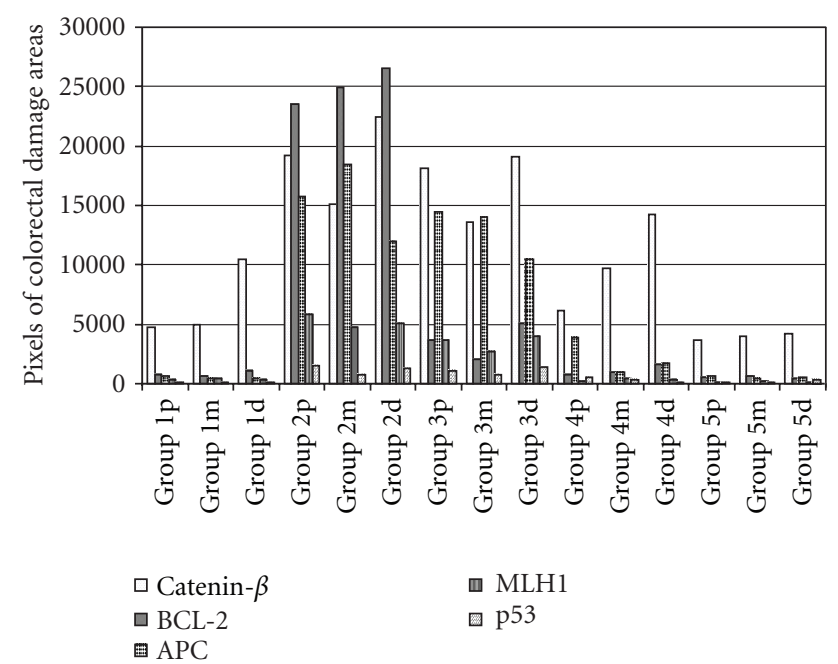

FIGURE 4: Image analysis of colorectal damaged area observed in the three portions of the colon in each experimental mice group. The quantification in pixels of the immunoreactive area affected was performed by a software analysis of the colorectal sections studied. Group 5 showed a significantly difference $(P<0.05)$ to the other treatment groups (3 and 4).

FR91 alone during the entire experimental study. The data obtained suggests that FR91 may be an important chemopreventive agent against intestinal inflammation in mice colon. In our experiment, we induced a wide range of colorectal lesions to better evaluate the effects of an anticancer agent (FR91) on chronic ulcerative colitis in mice. The repeated administration of DSS as an inductor of ulcerative lesions in mice models of colitis was extensively reported [1318], being essential in advancing our understanding of the complex interactions between the environment, genetics, and epithelial barrier dysfunction in the human-related Inflammatory Bowel Disease [19-22]. In this study, the use of DSS in drinking water during five weeks resulted in epithelial damage and a robust inflammatory response, obtaining a valid mice model acute injury colon to test an eight-week treatment with FR91, as reported in previous studies [2326]. Our results demonstrated that the optimal dose response was the 20\% FR91 concentration tested in mice group 5 , where no histological alterations or mild lesions were observed. Routinary histological staining [20, 23, 24, 27] and pretumoral cell markers $[18,20,23,28]$ used to evaluate the severity of lesions confirmed the protective effect of FR91

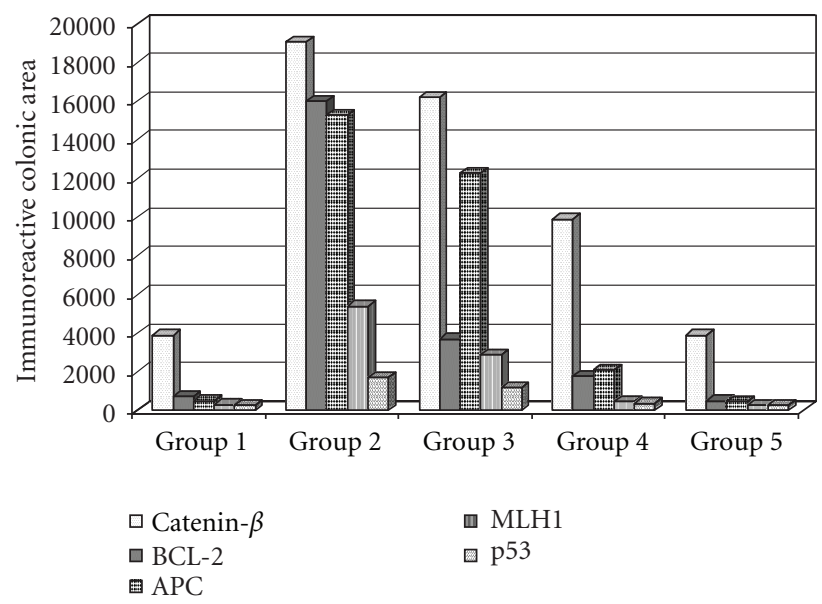

FIGURE 5: Image analysis of the mean value of colorectal damage area marked by immunoreactivity in mice groups. Group 5 showed a significantly difference $(P<0.05)$ to the other treatment groups ( 3 and 4 ), and a similar mean value when compared with negative control (group 1).

against the inflammation effect of DSS. These pretumoral cell markers such as APC, p53, MLH, BCL-2, Catenin- $\beta$, and cytokines, among others, take part in the molecular pathogenesis pathway of chronical colorectal inflammation that has been reported to derive at further stages in the development of sporadic colorectal carcinoma and colitisassociated colon cancer [22, 29-31]. The present results show the same significant interaction of these genetic markers in the pathologic characterization of the DSS-induced lessions observed. As reported in similar studies, a dosis-dependent effect is normally associated with the chemoprevention process in DSS-induced colitis $[25,26]$. Therefore, we tested other lower FR91 concentrations in drinking water, obtaining gradual colorectal lesions as the FR91 concentrations decreased. Moreover, we also tested the effects of FR91 in mice during a large period of time ( 8 weeks), obtaining similar histological results as the nontreated mice, which indicates a chemopreventive action against carcinogenesis without interfering with healthy epithelial structures of the mice colon.

Chemopreventive effects of a wide range of compounds on colonic tumors induced in mice have been reported previously, such as organosulfur compounds [25], N acetylcysteine [32], tetrandrine [33], phytosteryl ferulates [29], 
and dibenzoylmethane derivatives [34]. The DSS-induced lessions reported in these studies are similar to that observed in the preset work, where generally predominated in the midcolon $[7,35]$ and distal $[24,36]$ intestinal portions. These particular locations have been attributed to several factors such as the selective uptake of DSS in certain colonic portions, the presence of weak intestinal barrier regions, and different distribution of macrophages populations along the mice colon.

Alterations in the production of many cytokines have been observed in patients with IBD [37]. Although the real impact of these findings in the pathogenesis of IBD remains a controversial issue, as it is unclear whether they are primary or secondary factors involved in the regulation of the mucosal intestinal immune system, they can be considered as markers in the differentiation of groups of patients. In active IBD, an unbalance between regulatory and effector cells has been described, which mainly involves effector $\mathrm{T}$ cells (Th1 and Th2) and regulatory T cells (Tregs, Th3). CD is associated with a Th1 T-cell cytokine profile, including IFN- $\gamma$, TNF- $\alpha$, and IL-12, whereas UC is associated with a modified Th2 type response cytokine profile including IL-15 and IL-10 [38]. In addition, these findings have been recently complemented by the discovery of the IL-23/IL-17 axis, that is, part of the effector T-cell immunological response and seems to be involved in IBD. Levels of expression of IL-23 and IL-17 are increased in patients with active IBD [39].

Although specific determination of the FR91 metabolic action in the colitis prevention and treatment will require further investigations, we showed that FR91 prevented ulcerative lesions in mice models of colitis, inhibiting the development of colorectal tumors. FR91 has proved to be an interesting and promising investigational agent for studying chemoprevention of carcinogenesis.

\section{Abbreviations}

$\begin{array}{ll}\text { CL: } & \text { Crypt of Lieberkuhn } \\ \text { d: } & \text { Distal portion of the colon } \\ \text { GI: } & \text { Intestinal glands } \\ \text { Gr 1-5: } & \text { Group 1s5 } \\ \text { LP: } & \text { Lamina propria } \\ \text { m: } & \text { Middle portion of the colon } \\ \text { MM: } & \text { Muscularis mucosa } \\ \text { p: } & \text { Proximal portion of the colon } \\ \text { TM: } & \text { Tunica muscularis externa } \\ \text { TS: } & \text { Tunica serosa. }\end{array}$

\section{References}

[1] J. Keohane, C. O’Mahony, L. O’Mahony, S. O’Mahony, E. M. Quigley, and F. Shanahan, "Irritable bowel syndrome-type symptoms in patients with inflammatory bowel disease: a real association or reflection of occult inflammation," American Journal of Gastroenterology, vol. 105, no. 8, pp. 1789-1794, 2010.

[2] F. Scaldaferri and C. Fiocchi, "Inflammatory bowel disease: progress and current concepts of etiopathogenesis," Journal of Digestive Diseases, vol. 8, no. 4, pp. 171-178, 2007.
[3] N. Harpaz and A. D. Polydorides, "Colorectal dysplasia in chronic inflammatory bowel disease: pathology, clinical implications, and pathogenesis," Archives of Pathology and Laboratory Medicine, vol. 134, no. 6, pp. 876-895, 2010.

[4] I. Dotan, "Disease behavior in adult patients: are there predictors for stricture or fistula formation?" Digestive Diseases, vol. 27, no. 3, pp. 206-211, 2009.

[5] C. O. Elson, R. B. Sartor, G. S. Tennyson, and R. H. Riddell, "Experimental models of inflammatory bowel disease," Gastroenterology, vol. 109, no. 4, pp. 1344-1367, 1995.

[6] I. Okayasu, S. Hatakeyama, M. Yamada, T. Ohkusa, Y. Inagaki, and R. Nakaya, "A novel method in the induction of reliable experimental acute and chronic ulcerative colitis in mice," Gastroenterology, vol. 98, no. 3, pp. 694-702, 1990.

[7] H. S. Cooper, S. N. Murthy, R. S. Shah, and D. J. Sedergran, "Clinicopathologic study of dextran sulfate sodium experimental murine colitis," Laboratory Investigation, vol. 69, no. 2, pp. 238-249, 1993.

[8] J. Berdy, "Bioactive microbial metabolites: a personal view," Journal of Antibiotics, vol. 58, no. 1, pp. 1-26, 2005.

[9] D. J. Newman and G. M. Cragg, "Natural products as sources of new drugs over the last 25 years," Journal of Natural Products, vol. 70, no. 3, pp. 461-477, 2007.

[10] C. Olano, C. Méndez, and J. A. Salas, "Antitumor compounds from actinomycetes: from gene clusters to new derivatives by combinatorial biosynthesis," Natural Product Reports, vol. 26, no. 5, pp. 628-660, 2009.

[11] A. L. Demain and S. Sanchez, "Microbial drug discovery: 80 Years of progress," Journal of Antibiotics, vol. 62, no. 1, pp. 5$16,2009$.

[12] V. R. Lombardi, E. Martínez, R. Chacn, I. Etcheverría, and R. Cacabelos, "Effects of FR-91 on immune cells from healthy individuals and from patients with non-hodgkin lymphoma," Journal of Biomedicine and Biotechnology, vol. 2009, Article ID 187015, 9 pages, 2009.

[13] J. Y. Cho, H. J. Chang, S. K. Lee, H. J. Kim, J. K. Hwang, and H. S. Chun, "Amelioration of dextran sulfate sodium-induced colitis in mice by oral administration of beta-caryophyllene, a sesquiterpene," Life Sciences, vol. 80, no. 10, pp. 932-939, 2007.

[14] Y. Araki, K. Mukaisyo, H. Sugihara, Y. Fujiyama, and T. Hattori, "Increased apoptosis and decreased proliferation of colonic epithelium in dextran sulfate sodium-induced colitis in mice," Oncology Reports, vol. 24, no. 4, pp. 869-874, 2010.

[15] T. C. Huang, S. S. Tsai, L. F. Liu, Y. L. Liu, H. J. Liu, and K. P. Chuang, "Effect of Arctium lappa L. in the dextran sulfate sodium colitis mouse model," World Journal of Gastroenterology, vol. 16, no. 33, pp. 4193-4199, 2010.

[16] B. O. Lim, S. Y. Choi, S. J. Hur et al., "Anti-inflammatory effects of inonotus obliquus in colitis induced by dextran sodium sulfate," Journal of Biomedicine and Biotechnology, vol. 2010, Article ID 943516, 5 pages, 2010.

[17] C. G. Whittem, A. D. Williams, and C. S. Williams, "Murine colitis modeling using dextran sulfate sodium (DSS)," Journal of Visualized Experiments, vol. 19, no. 35, p. 1652, 2010.

[18] T. Tanaka, H. Kohno, R. Suzuki et al., "Dextran sodium sulfate strongly promotes colorectal carcinogenesis in $\mathrm{Apc}^{\mathrm{Min} /+}$ mice: inflammatory stimuli by dextran sodium sulfate results in development of multiple colonic neoplasms," International Journal of Cancer, vol. 118, no. 1, pp. 25-34, 2006.

[19] E. H. Birkenmeier, A. Torrey, and J. P. Sundberg, "Chromosomal location of modifier genes determining sensitivity of mice to dextran sulphate sodium," in Inflammatory Bowel Disease, G. Tytgat, J. Bartelsman, and S. van Deventer, Eds., pp. 401407, Boston, Mass, USA, 1995. 
[20] T. Tanaka, "Colorectal carcinogenesis: review of human and experimental animal studies," Journal of Carcinogenesis, vol. 8, article 5, 2009.

[21] S. Nell, S. Suerbaum, and C. Josenhans, "The impact of the microbiota on the pathogenesis of IBD: lessons from mouse infection models," Nature Reviews Microbiology, vol. 8, no. 8, pp. 564-577, 2010.

[22] T. A. Ullman and S. H. Itzkowitz, "Intestinal inflammation and cancer," Gastroenterology, vol. 140, no. 6, pp. 1807-1816, 2011.

[23] J. G. Wang, D. F. Wang, B. J. Lv, and J. M. Si, "A novel mouse model for colitis-associated colon carcinogenesis induced by 1, 2-dimethylhydrazine and dextran sulfate sodium," World Journal of Gastroenterology, vol. 10, no. 20, pp. 2958-2962, 2004.

[24] M. Mähler, I. J. Bristol, E. H. Leiter et al., "Differential susceptibility of inbred mouse strains to dextran sulfate sodiuminduced colitis," American Journal of Physiology, vol. 274, no. 3, pp. G544-G551, 1998.

[25] H. Sumiyoshi and M. J. Wargovich, "Chemoprevention of 1, 2dimethylhydrazine-induced colon cancer in mice by naturally occurring organosulfur compounds," Cancer Research, vol. 50, no. 16, pp. 5084-5087, 1990.

[26] D. L. Dillehay, S. K. Webb, E. M. Schmelz, and A. H. Merrill, "Dietary sphingomyelin inhibits 1, 2-dimethylhydrazineinduced colon cancer in CF1 mice," Journal of Nutrition, vol. 124, no. 5, pp. 615-620, 1994.

[27] M. Darsigny, J. P. Babeu, A. A. Dupuis et al., "Loss of hepatocyte-nuclear-factor- $4 \alpha$ affects colonic ion transport and causes chronic inflammation resembling inflammatory bowel disease in mice," Plos One, vol. 4, no. 10, Article ID e7609, 2009.

[28] S. M. Cruickshank, N. R. English, P. J. Felsburg, and S. R. Carding, "Characterization of colonic dendritic cells in normal and colitic mice," World Journal of Gastroenterology, vol. 11, no. 40, pp. 6338-6347, 2005.

[29] M. S. Islam, T. Murata, M. Fujisawa et al., "Anti-inflammatory effects of phytosteryl ferulates in colitis induced by dextran sulphate sodium in mice," British Journal of Pharmacology, vol. 154, no. 4, pp. 812-824, 2008.

[30] J. Y. Xiang, L. G. Wu, X. L. Huang et al., "Amelioration of murine dextran sulfate sodium-induced colitis by nuclear factor- $\kappa \mathrm{b}$ decoy oligonucleotides," American Journal of Surgery, vol. 197, no. 6, pp. 797-805, 2009.

[31] D. K. Zhang, L. N. Cheng, X. L. Huang, W. Shi, J. Y. Xiang, and H. T. Gan, "Tetrandrine ameliorates dextran-sulfate-sodiuminduced colitis in mice through inhibition of nuclear factor- $\kappa \mathrm{B}$ activation," International Journal of Colorectal Disease, vol. 24, no. 1, pp. 5-12, 2009.

[32] Y. You, J. J. Fu, J. Meng, G. D. Huang, and Y. H. Liu, "Effect of $\mathrm{N}$-acetylcysteine on the murine model of colitis induced by dextran sodium sulfate through up-regulating PON1 activity," Digestive Diseases and Sciences, vol. 54, no. 8, pp. 1643-1650, 2009.

[33] D. K. Zhang, L. N. Cheng, X. L. Huang, W. Shi, J. Y. Xiang, and H. T. Gan, "Tetrandrine ameliorates dextran-sulfate-sodiuminduced colitis in mice through inhibition of nuclear factor- $\kappa \mathrm{B}$ activation," International Journal of Colorectal Disease, vol. 24, no. 1, pp. 5-12, 2009.

[34] R. Murakami, M. Uchida, O. Hori et al., "Efficacy of dibenzoylmethane derivatives in protecting against endoplasmic reticulum stress and inhibiting nuclear factor kappa B on dextran sulfate sodium induced colitis in mice," Biological and Pharmaceutical Bulletin, vol. 33, no. 12, pp. 2029-2032, 2010.
[35] A. Minocha, C. Thomas, and R. Omar, "Lack of crucial role of mast cells in pathogenesis of experimental colitis in mice," Digestive Diseases and Sciences, vol. 40, no. 8, pp. 1757-1762, 1995.

[36] S. Tokoi, T. Ohkusa, I. Okayasu, and K. Nakamura, "Population changes in immunoglobulin-containing mononuclear cells in dextran sulfate sodium-induced coltitis," Journal of Gastroenterology, vol. 31, no. 2, pp. 182-188, 1996.

[37] T. Yamamoto, S. Umegae, T. Kitagawa, and K. Matsumoto, "Systemic and local cytokine production in quiescent ulcerative colitis and its relationship to future relapse: a prospective pilot study," Inflammatory Bowel Diseases, vol. 11, no. 6, pp. 589-596, 2005.

[38] S. Danese and C. Fiocchi, "Etiopathogenesis of inflammatory bowel diseases," World Journal of Gastroenterology, vol. 12, no. 30, pp. 4807-4812, 2006.

[39] O. H. Nielsen, I. Kirman, N. Rüdiger, J. Hendel, and B. Vainer, "Upregulation of interleukin-12 and -17 in active inflammatory bowel disease," Scandinavian Journal of Gastroenterology, vol. 38, no. 2, pp. 180-185, 2003. 


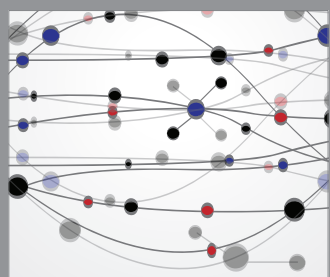

The Scientific World Journal
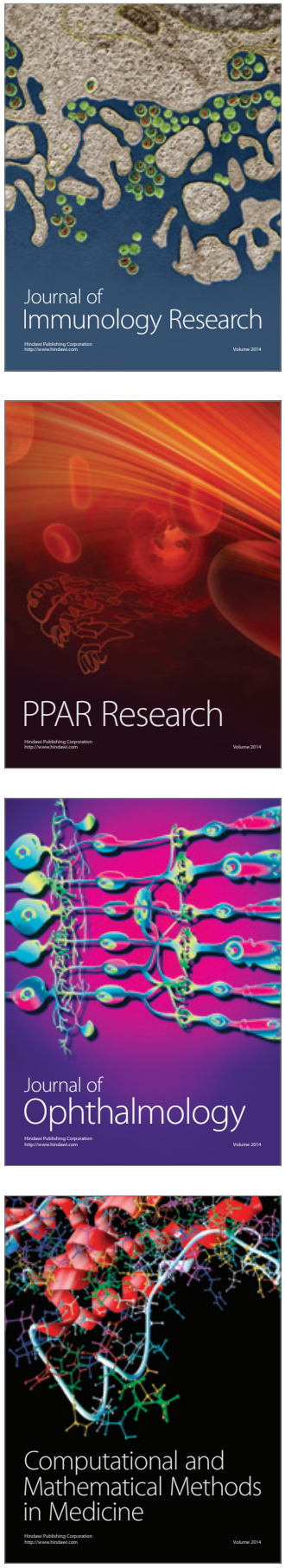

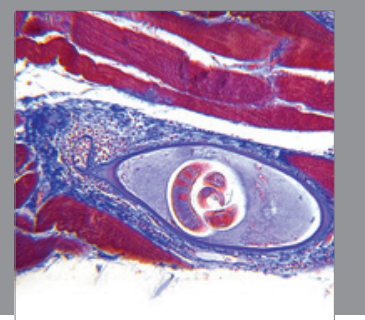

Gastroenterology

Research and Practice
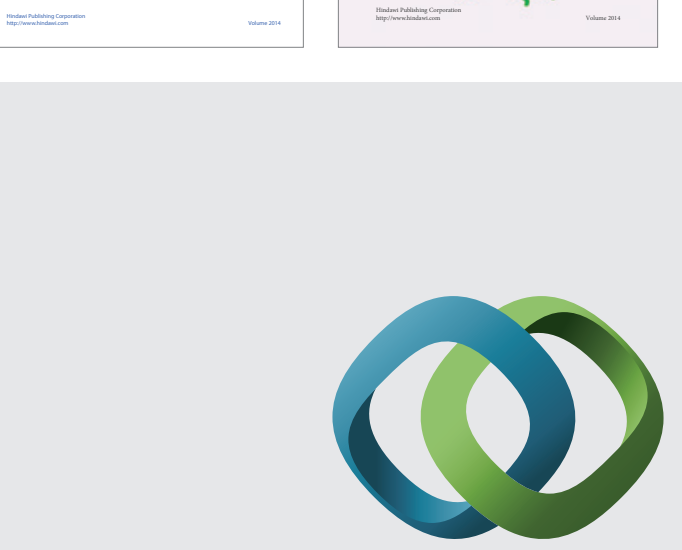

\section{Hindawi}

Submit your manuscripts at

http://www.hindawi.com
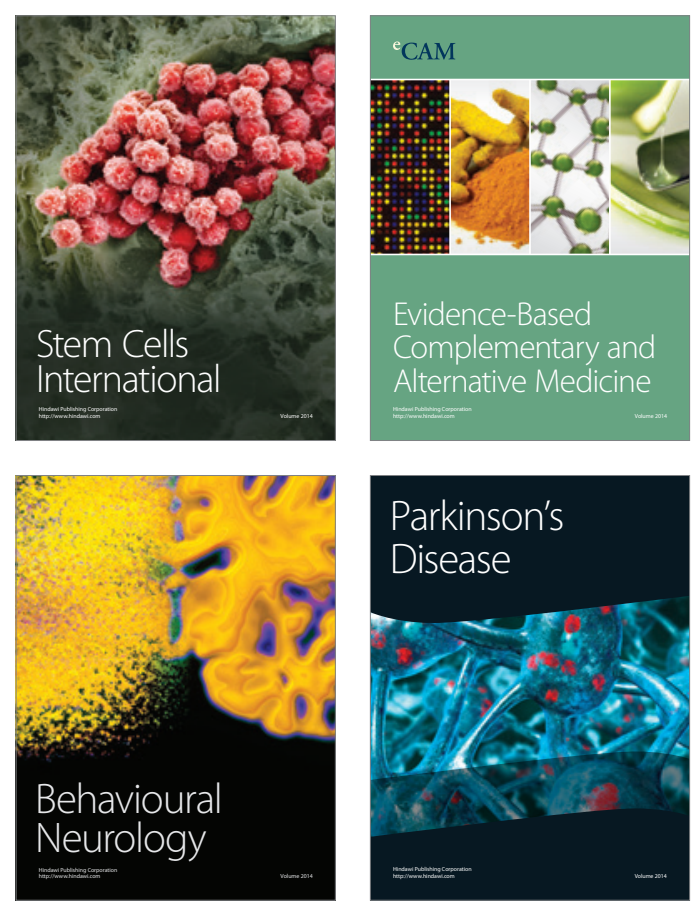

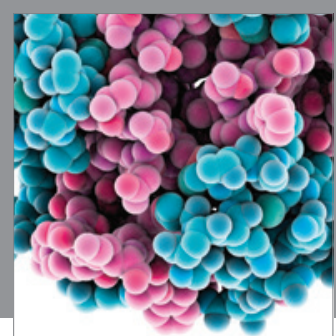

Journal of
Diabetes Research

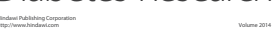

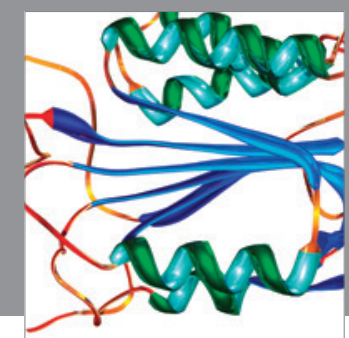

Disease Markers
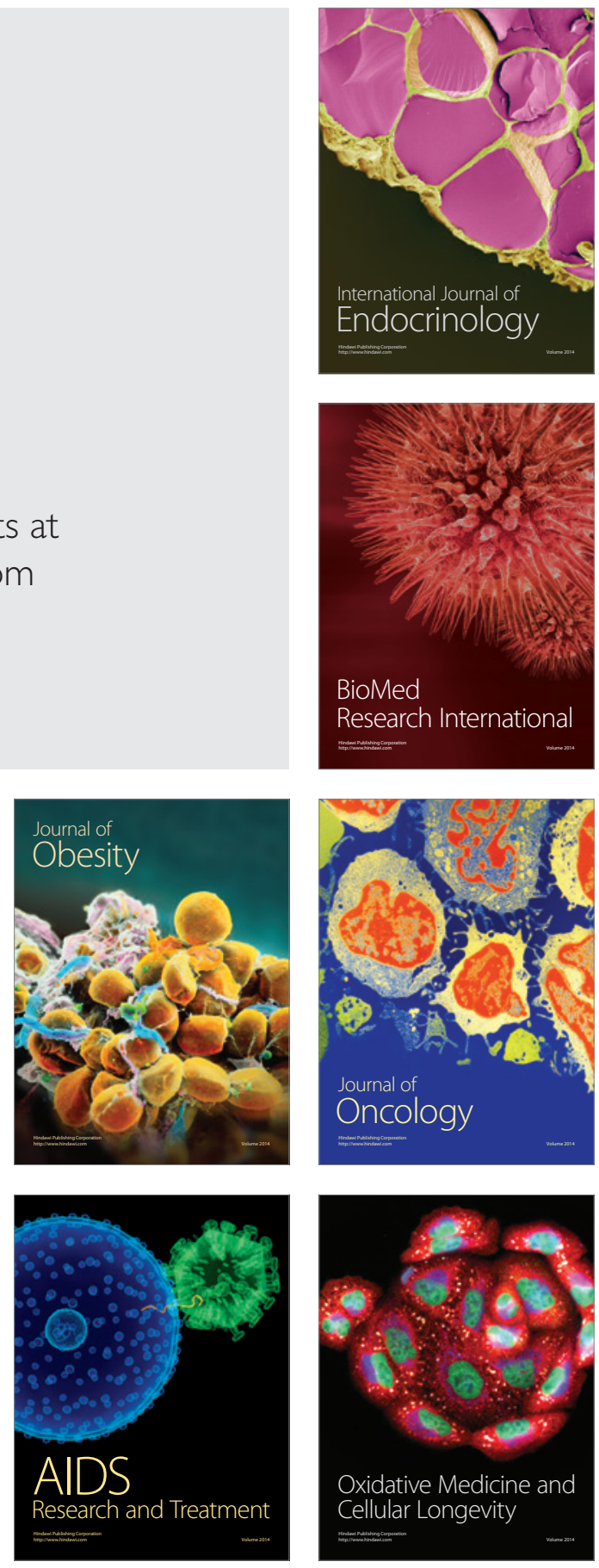